\title{
tic\&société
}

Vol. 8, N 1-2 | 1er semestre 2014 et 2ème semestre 2014

Quelles perspectives critiques pour aborder les TIC ?

\section{Médias sociaux, idéologie invisible et réel : pour une dialectique du concret}

Maxime OUELLET, André MONDOUX and Marc MENARD

\section{(2) OpenEdition \\ Journals}

\section{Electronic version}

URL: http://journals.openedition.org/ticetsociete/1391

DOI: 10.4000/ticetsociete.1391

\section{Publisher}

Association ARTIC

\section{Electronic reference}

Maxime OUELLET, André MONDOUX and Marc MENARD, « Médias sociaux, idéologie invisible et réel pour une dialectique du concret », tic\&société [Online], Vol. 8, N 1-2। 1er semestre 2014 et 2ème semestre 2014, Online since 30 May 2019, connection on 10 December 2020. URL : http:// journals.openedition.org/ticetsociete/1391 ; DOI : https://doi.org/10.4000/ticetsociete.1391 
tic\&société - 8 (1-2), 2014

\title{
Médias sociaux, idéologie invisible et réel : pour une dialectique du concret
}

\author{
Maxime OUELLET \\ École des médias \\ CRICIS et GRISQ \\ UQAM \\ ouellet.maxime@uqam.ca
}

André MONDOUX

École des médias

CRICIS et GRISQ

UQAM

mondoux.andre@uqam.ca

Marc MENARD

École des médias

CRICIS et GRISQ

UQAM

menard.marc@ugam.ca 


\title{
Médias sociaux, idéologie invisible et réel : pour une dialectique du concret
}

\begin{abstract}
Maxime OUELLET est professeur à l'École des médias de l'Université du Québec à Montréal (UQAM). II est également chercheur au Centre de recherche interuniversitaire sur la communication, l'information et la société (CRICIS) et au Groupe de recherche sur l'information et la surveillance au quotidien (GRISQ). Ses intérêts de recherche portent sur le rôle des médias dans la mondialisation et se situent à la rencontre de l'économie politique de la communication et des Cultural Studies. II s'intéresse plus particulièrement au domaine de l'économie politique culturelle, notamment à la question de la création de la valeur dans les médias sociaux et à la banalisation de la surveillance.
\end{abstract}

André MONDOUX est professeur à l'École des médias de l'Université du Québec à Montréal (UQAM) et est co-fondateur du Groupe de recherche sur l'information et la surveillance au quotidien (GRISQ) ainsi que chercheur au Centre de recherche interuniversitaire sur la communication, l'information et la société (CRICIS). Docteur en sociologie, il se spécialise dans les problématiques relevant des rapports entre société, technique et individuation collective. À ce titre, il a publié plusieurs articles portant sur les médias socionumériques et leur apport aux dynamiques d'ordre politico-idéologique et à la (re)production sociale, de même que sur leur intégration aux processus de banalisation de la surveillance qui caractérisent les sociétés dites postmodernes. Ses recherches les plus récentes portent sur la numérisation des processus de médiation symbolique (la 'machinisation' du tiers symbolique gouvernance algorithmique) et ses liens sociopolitiques et économiques avec le phénomène du Big Data, plus précisément comment les rapports de pouvoir et de communication sont affectés par la dynamique de l'intégration des informations produites par les médias socionumériques dans des processus de cueillette et d'analyse de quantités massives de données. II s'intéresse également aux nouvelles plateformes mobiles et les nouveaux circuits de marchandisation de l'information qui s'inscrivent dans des formes de surveillance continue désormais constitutive de liens sociaux.

Marc MENARD détient un doctorat en science économique de l'Université Paris VIII. II est professeur à l'École des médias de l'Université du Québec à Montréal (UQAM), membre du Groupe de recherche sur l'information et la surveillance au quotidien (GRISQ) et directeur par interim du Centre de recherche 


\section{Maxime OUELLET, André MONDOUX et Marc MENARD}

interuniversitaire sur la communication, l'information et la société (CRICIS). Ses travaux portent sur l'économie de la culture, les industries culturelles et les nouvelles formes de marchandisation de l'information et de la communication. II a publié en 2001 Les chiffres des mots. Portrait économique du livre au Québec et, en 2005, Éléments pour une économie des industries culturelles.

Résumé : Cet article aborde les TIC, plus spécifiquement les médias socionumériques, dans une perspective critique. Nous soutenons que, pour aborder les TIC dans une perspective critique, il est nécessaire de réhabiliter un certain nombre de notions fondatrices de la tradition de la théorie critique, c'està-dire les concepts de totalité, d'idéologie, de dialectique, de fétichisme et d'aliénation. Au contraire des approches qui postulent que nous entrons dans l'ère de la fin des idéologies et de la représentation de la société comme totalité, nous avançons qu'une théorie critique des médias socionumériques se doit d'analyser leurs usages dans le contexte des transformations sociétales plus profondes qui ont cours au sein des sociétés capitalistes. Ces transformations, notamment la globalisation capitaliste, s'inscrivent dans une dynamique de totalisation, qui a entre autres été rendue possible grâce au déploiement des TIC. Selon nous, ce n'est que lorsque la technique aura réintégré le terrain idéologique qu'elle redeviendra sujette à débat et, de ce fait, réellement appropriable.

Mots-clés : TIC, Médias sociaux, théorie critique, idéologie, surveillance.

Abstract : This article discusses ICTs, specifically social media, from a critical perspective. We maintain that doing so necessitates the rehabilitation of certain foundational concepts such as dialectics, ideology, fetishism and alienation. In contrast to claims of the end of both ideology and of the representation of society as a totality, we posit that a critical theory of social media must analyze its uses in the context of profound social changes in capitalist societies. These transformations, including capitalist globalization, are constituents of a dynamic of totalization, which is made possible by the deployment of ICTs. In our view, it is only when technique is reintegrated with the ideological terrainthat it will be once again subject to debate and, therefore, truly appropriable.

Keywords : ICTs, social media, critical theory, ideology, surveillance.

Resumen : Este artículo aborda las TIC (Tecnologías de la Información y de la Comunicación), y más específicamente los medios de comunicación sociodigitales, bajo una perspectiva crítica. Para ello resulta necesario rehabilitar 
Médias sociaux, idéologie invisible et réel : pour une dialectique du concret

algunas nociones situadas en la tradición de la teoría crítica, como son los conceptos de totalidad, de ideología, de dialéctica, de fetichismo y de alienación. Al contrario de las teorías que estipulan que entramos en la era del fin de las ideologías y de la representación de la sociedad como una totalidad, se propone la idea de que una teoría crítica de los medios de comunicación socio-digitales debe analizar los usos de estos medios en el contexto de las transformaciones sociales más profundas que tienen lugar en el seno de la sociedad capitalista. Estas transformaciones, particularmente la globalización capitalista, se inscriben en una dinámica de totalización que ha sido posible gracias al despliegue de las TIC. Se concluye que únicamente cuando al lado de la técnica se considere el terreno ideológico y que por tanto la técnica sea objeto de debate nos encontraremos con una técnica que puede ser realmente apropiada.

Palabras clave: TIC, Medios de comunicación sociales, teoría critica, ideología, vigilancia. 
Maxime OUELLET, André MONDOUX et Marc MENARD

\section{Introduction}

Le terme "social 》 accolé aux médias socionumériques (MSN) n'est pas innocent. Contrairement aux outils de communication (télévision, téléphone, radio, presse, etc.), chacun tout aussi « social » que les autres, les MSN sont en effet investis de la prétention de " produire " du " social » et ce, directement à partir de l'usage - individuel - de la technique. Nous sommes ici face à une situation qui n'est pas sans évoquer ce que Heidegger nommait la "représentation anthropologico-instrumentale » de la technique: l'usage individuel d'un outil neutre permettant de révéler le réel. Appliquée au " social » (les MSN), cette notion se traduit par une dynamique marquée par le surdéterminisme d'un individu posé comme degré zéro du social ; espace d'un individu «libre " surgissant avant le social dont il serait l'unique producteur. Voilà bien la promesse des MSN : I'usage d'outils de communication comme fer de lance de l'émancipation (empowerment) des individus, émancipation comprise en tant que libération des instances institutionnelles. Sous cet angle, nulle surprise à ce que les MSN émergent dans la foulée des thèses sur la fin des grandes idéologies. Nous avons déjà explicité cette dynamique (Mondoux, 2009, 2011 et 2012) sous la notion d'hyperindividualisme ${ }^{1}$, soit l'émergence d'un sujet qui, refusant le déterminisme des instances morales, disciplinaires et idéologiques, prétend ultimement advenir par et pour lui-même. Recadrée à l'aune des MSN, cette dynamique repose sur la croyance que l'usage individuel de la technique permettrait de produire un social sans médiation esthéticosymbolico-idéologique; une croyance en cela conforme aux valeurs du néolibéralisme.

Ce nouveau statut de producteur du social place l'outil au cœur de la médiation symbolique. Cette dynamique sociohistorique que certains associent à une misère symbolique (Stiegler, 2004) ou encore à une substitution de la médiation symbolique par un système décisionnel-opérationnel (Freitag, 1986), soulève plusieurs questions : comment la technique en vient-elle à "épuiser » le symbolique ? Quel est son rôle dans la (re)production de l'ordre social ? Quel est son apport dans la reconduction du capitalisme dominant? Nous croyons que pour répondre à ces questions il est nécessaire d'aborder cette problématique par la dimension de l'idéologie puisque cette dernière est la grande absente de l'analyse des médias socionumériques, occultée par des

\footnotetext{
${ }^{1} \mathrm{Ne}$ pas confondre notre utilisation de la notion d'hyperindividualisme en tant que configuration socio-historique de rapports sociaux et approche épistémologique consistant à opposer les individus aux rapports sociaux globaux (la société) et qui, d'Élias à Bourdieu en passant par Luckmann, a été critiqué en tant qu'individualisme méthodologique.
} 
Médias sociaux, idéologie invisible et réel : pour une dialectique du concret

outils techniques présentés comme neutres ou «présociaux ». La technique est-elle réellement un moyen neutre - non idéologique - permettant d'accéder au réel ?

Les thèses affirmant la fin des idéologies et l'apparition d'un sujet pleinement émancipé, deux prétentions que l'on retrouve dans de nombreux discours portant sur l'usage des médias sociaux, reconduisent implicitement l'idée que le réel est désormais posé comme immédiatement accessible sans médiation symbolique, idéologique ou politique, comme l'atteste notamment l'actuelle fascination pour le data-mining: le forage extirpe des échantillons de réel, transformant du coup l'information en données objectives.

Dans cet article, nous soutenons au contraire que la technique, en se posant en substitut face à la médiation symbolique (la «production » du social par des " outils " comme Facebook et autres), participe d'une forme de domination en apparence non-idéologique. En effet, n'ayant plus à se légitimer sur le terrain de l'idéologie et du politique, ce discours dominant peut donc nourrir des prétentions ontologiques (se projeter comme le réel/le monde lui-même) et s'actualiser en une dynamique "immanente " pleinement vouée à sa propre efficience où les moyens deviennent une fin.

II faut prendre garde ici à ne pas tomber dans une dichotomie réductrice consistant à forcer le choix entre un sujet pleinement souverain et une technique quasi autonome ; entre une technique résolument individuelle et une dynamique sociale totalement hégémonique. En effet, l'on peut reprocher à Ellul de côtoyer de trop près l'idée d'une technique totalitaire. De même, si Heidegger a bel et bien vu la possibilité d'une aliénation par la technique, son approche " existentielle " laisse peu ou pas assez de place au social ; au contraire d'un Simondon qui lie effectivement technique et société, mais néglige la dimension de l'aliénation par la technique. Notre approche se situe dans la visée des approches dialectiques qui effectuent une synthèse entre les perspectives marxiennes et heideggériennes (Axelos, 1961; Vioulac, 2009, Fischbach 2011). Les relectures contemporaines de Marx à partir d'Heidegger permettent de comprendre comment la technique et l'individu s'inscrivent à l'intérieur d'une totalité où les deux termes sont unis dans un rapport de transduction, c'est-àdire créant une dynamique totalisante qui, à son tour, les (re)produit individuellement.

Dans un premier temps, nous verrons qu'il est nécessaire pour aborder les TIC dans une perspective critique de réhabiliter un certain nombre de notions fondatrices de la tradition de la théorie critique, c'est-à-dire les concepts de 


\section{Maxime OUELLET, André MONDOUX et Marc MENARD}

totalité, de dialectique, de fétichisme et d'aliénation. Ensuite, afin de montrer en quoi la soi-disant émancipation du sujet au moyen des médias socionumériques consiste en une forme d'aliénation, une analyse épistémologique et sociohistorique de la notion d'idéologie sera présentée. D'une part, sur le plan épistémologique, pour la réhabiliter en tant qu'incontournable médiation constitutive du rapport au réel. D'autre part, sur le plan sociohistorique, afin de dévoiler les fondements idéologiques d'une technique posée comme aidéologique et ainsi paver la voie à une véritable utilisation émancipatrice des médias socionumériques. Dans un troisième temps, nous verrons que l'usage des médias sociaux participe pleinement à la (re)production d'un sujet dont "l'émancipation" consiste en fait en une adaptation à la logique d'autovalorisation capitaliste notamment via les pratiques de surveillance mutuelle. En conclusion, nous soutiendrons que ce n'est que lorsque la technique aura réintégré le terrain idéologique qu'elle redeviendra " débattable » et de ce fait réellement appropriable.

\section{Médias socionumériques, théorie critique et totalité}

Pour aborder de manière critique les médias socionumériques, il importe de savoir ce qu'on entend par "social » dans le terme médias "sociaux". Le principal postulat sociologique sur lequel reposent les théories portant sur les usages des médias socionumériques est qu'on assisterait à la dissolution de la société comprise comme une totalité, laquelle se verrait remplacée par une société en réseaux (Castells, 2001) où la médiation au moyen des techniques de communication remplacerait les médiations symboliques qui ordonnançaient la pratique sociale dans les sociétés antérieures. Sur le plan théorique, il faudrait donc "cesser de penser la société comme une unité, comme une structure capable de tout intégrer. L'image de la société est donc floue, non seulement parce que celle-ci bouge tout le temps, mais aussi parce qu'elle n'a plus d'unité » (Jauréguiberry et Proulx, 2011, p. 104). Dans ce contexte, "la seule réponse possible consiste à penser la société non plus comme une unité donnée et structurante, mais comme un résultat éphémère et aléatoire de pratiques sociales" (Idem, p. 105). Posé comme fondamentalement inatteignable dans sa totalité, le social se réduirait ainsi à sa dimension "locale » ou "concrète ", ce qui explique en partie le retour en force de la notion de " communauté » au sein de l'univers des médias socionumériques.

La notion de "communauté ", produite par des "gestionnaires de communautés ", repose sur la présupposition d'une société formée de réseaux qui se structurent et se déstructurent de manière contingente au moyen de la 
Médias sociaux, idéologie invisible et réel : pour une dialectique du concret

médiation d'objets techniques. Cette représentation du social sans totalité est développée théoriquement de la façon la plus cohérente par la théorie des acteurs-réseaux (Latour, 1994). Selon cette approche, il faudrait partir des interactions réticulées entre les acteurs et les objets techniques pour expliquer la stabilisation contingente et partielle que prend le social. La théorie des acteurs-réseaux nie donc les principes fondamentaux de la sociologie classique qui reposent sur la dynamique d'intersubjectivité au fondement du social, pour lui substituer celle de l'inter-objectivité. L'analyse sociologique devrait donc accepter une certaine dose de fétichisme puisque la technique possèderait selon cette approche un statut quasi-ontologique. En clair, tout comme les médias socionumériques auraient le pouvoir de produire du social : "Les objets font quelque chose, ils ne sont pas seulement les écrans ou les rétroprojecteurs de notre vie sociale. Leur seule fonction n'est pas de "blanchir " l'origine sociale des forces que nous projetons sur eux». (Idem, p. 49) Selon Latour : "Les sociologues ne chercheraient-ils pas midi à 14 heures en construisant le social avec du social ou en maçonnant ses fissures avec du symbolique, alors que les objets sont omniprésents dans toutes les situations dont ils cherchent le sens? » (Ibid., p. 46). Contre la sociologie critique qui insiste sur la notion de domination, la théorie des acteurs-réseaux cherche à montrer la capacité (l'empowerment) des acteurs à se brancher et à se rebrancher dans une multitude de connexions possibles au moyen des outils techniques. Nous verrons plus loin que ce type de discours théorique qui pose les individus comme ontologiquement premier, déliés de toute institution sociale grâce à leurs interactions dans des réseaux techniques, correspond à une forme de subjectivité qui participe activement à la reproduction du capitalisme avancé ${ }^{2}$. (Vandenberghe, 2006)

Mais, au-delà de la question de la subjectivité sociale, nous nous attarderons premièrement à la notion de totalité et à sa prétendue disparition. Selon nous, le principal problème des analyses qui postulent la disparition de la totalité est qu'elles amalgament la totalité avec l'une des formes qu'elle a prise dans I'histoire, dans ce cas-ci l'État-nation qui est au fondement de la représentation de la totalité dans la modernité classique. Une perspective critique devrait être en mesure d'analyser de quelle manière les transformations sociétales plus profondes qui ont cours au sein des sociétés capitalistes avancées s'inscrivent dans une dynamique de totalisation, notamment la globalisation qui a entre autres été rendue possible grâce au déploiement des TIC (Freitag, 2008; Vioulac, 2012). L'abandon de la notion de totalité dans les sciences sociales en général, et dans le domaine des études en communication en particulier,

\footnotetext{
${ }^{2}$ À ce sujet voir également Labelle, 2007.
} 


\section{Maxime OUELLET, André MONDOUX et Marc MENARD}

conduit à l'impossibilité même de postuler une théorie critique de la société3. Comme le souligne Frank Fishbach à propos de la théorie critique :

"Son exigence de cohérence avec elle-même conduit la critique menée par la philosophie sociale à prendre la forme non pas d'une critique partielle et extérieure, mais celle d'une critique totalisante et immanente, par où nous entendons une critique qui ne s'appuie pas sur un élément abstraitement isolé de l'ensemble, mais une critique qui vise le cœur même de la société critiquée, à partir duquel on peut à la fois la saisir dans la totalité qu'elle forme, et rendre compte de la possibilité de la formation en elle d'un point de vue critique. » (2009, p. 147).

Dans la tradition de la théorie critique, qui puise ses sources notamment chez Hegel et Marx, la notion de totalité vise à intégrer l'analyse des faits particuliers dans une dynamique sociale plus large: "Par conséquent, dans l'emploi de la méthode théorique aussi, il faut que le sujet, la société, reste constamment présent à l'esprit comme donnée première. » (Marx, 1857, p. 10) La totalité ne se réduit donc pas en une accumulation de faits isolés qui seraient immédiatement perceptibles à partir d'une analyse strictement empirique. Reprenant les postulats méthodologiques hégéliano-marxien, Karel Kosik soutient qu'un des principes fondamentaux de la méthode dialectique est d'analyser les phénomènes concrets à partir de l'abstraction. La tâche de la dialectique est d'abolir le «pseudo-concret» dans lequel est enfermée la pensée non-réflexive pour en arriver à une compréhension adéquate de la totalité : "L'ensemble n'est pas immédiatement connaissable pour l'homme, même s'il lui est donné immédiatement dans la perception, la sensation et l'expérience. L'ensemble perçu directement est chaotique et obscur. Pour l'appréhender et le comprendre, l'analyser et l'expliquer, l'homme doit accomplir un détour : le concret devient compréhensible grâce à la médiation de l'abstrait, et l'ensemble grâce à la médiation des parties » $(1988$, p. 17).

C'est pourquoi l'un des fondateurs de la théorie critique, Max Horkheimer, distingue deux types de théorie : la théorie traditionnelle et la théorie critique. La théorie traditionnelle se limite à l'analyse des faits empiriques et observables, à partir desquels elle tente de formuler une série d'hypothèses qui donnent lieu à une théorie. Le problème de ce type d'analyse qui se contente d'analyser les faits isolément est qu'elle incapable de comprendre comment la réalité est le

\footnotetext{
${ }^{3}$ Pour une analyse de la notion de totalité au sein des études en communication, voir Voirol, 2008.
} 
Médias sociaux, idéologie invisible et réel : pour une dialectique du concret

produit d'une praxis, c'est-à-dire d'une pratique social-historique qui participe à la reproduction et à la transformation de la totalité. Au contraire des approches partielles et partiales, selon Horkeimer, " [la] théorie critique de la société, elle, prend pour objet les hommes en tant que producteurs de la totalité des formes que leur vie revêt dans l'histoire », (1970, p. 82).

La méthode dialectique cherche à montrer que la réalité est duale : il s'agit d'une totalité à la fois symbolique et matérielle. Elle vise à déchiffrer l'essence sociale qui se cache derrière la forme phénoménale des choses. Le concept d'«essence " est compris ici en termes social-historique, et non au sens transhistorique tel qu'utilisé dans la philosophie classique. La théorie critique montre notamment qu'une des spécificités du capitalisme est qu'il possède une essence qui est fondée sur une pratique social-historique, le travail abstrait. C'est le travail, en tant que médiation sociale spécifique au capitalisme, qui en constitue l'essence, laquelle interagit de manière dialectique avec les représentations sociales (Postone, 2009). C'est pourquoi Marx débutera son analyse dans Le Capital par la catégorie de la marchandise dont la réalité se présente de manière duale; valeur d'usage et valeur d'échange, lesquelles renvoient à la dualité du travail concret et du travail abstrait, ou encore au capital fixe et au capital variable (1993 [1867]). La marchandise correspond donc à la médiation sociale centrale qui est spécifique à la forme socialhistorique qu'est le capitalisme. En tant que chose sensible et suprasensible, elle est matérielle et symbolique; à la fois abstraite en tant que valeur ${ }^{4}$, et concrète en tant que valeur d'usage. En clair, la forme-valeur de la marchandise correspond à une norme qui régule les pratiques et les formes de conscience sociale; il s'agit d'un fait social total.

Sur le plan symbolique, le fétichisme de la marchandise est au fondement de l'imaginaire social du capitalisme (Castoriadis, 1975). II s'agit d'une projection qui se sépare du réel, mais qui n'en est pas moins plus réelle que le réel, puisqu'il permet la représentation de l'irreprésentable, par exemple, la réification de l'individu en force de travail ou encore la substitution de la richesse réelle par la valeur abstraite (l'argent). Le symbolique a pour fonction de rendre commensurables des choses qui en soi ne le sont pas. Bref, puisque le symbolique est nécessaire pour que l'échange social soit possible, la marchandise correspond au mode de symbolisation (Goux, 1973) qui est au fondement du mode d'objectivation purement quantitatif propre à la société

\footnotetext{
${ }^{4}$ II ne faut pas confondre valeur et valeur d'échange puisque cette dernière catégorie renvoie à la manière dont s'exprime la valeur d'une marchandise dans une autre. La catégorie de la valeur renvoie quant à elle à l'objectivation du travail abstrait. À ce sujet voir Tran Hai Hac, 2003.
} 


\section{Maxime OUELLET, André MONDOUX et Marc MENARD}

capitaliste. La marchandise organise donc les catégories et les cadres conceptuels de ce qui peut être imaginé (le réel); elle est l'instance apriorique qui fournit le sens à la pratique sociale et à l'identité des sujets.

C'est à partir du fétichisme de la marchandise comme fait social total qu'il faut comprendre l'analyse heideggérienne de la domination opérée par la technique dans le monde moderne (Fischbach, 2011). Tout comme le fétiche nous présente un monde inversé en ce que la production de marchandise a comme finalité leur valeur d'échange plutôt que la satisfaction des besoins humains, la technique n'est plus au service des finalités humaines, mais elle tend plutôt à servir à la reproduction de la valeur qui est devenue la nouvelle fin de la pratique humaine. La pratique humaine ne disparaît pas, mais son énergie vitale n'est plus consacrée à l'approfondissement de son expérience du monde à travers la médiation des formes symboliques et culturelles; l'expérience humaine est mise au service d'une technique elle-même arrimée à une économie autonomisée. La praxis se trouve donc marginalisée puisque ce n'est plus elle qui se donne par elle-même ses fins, c'est plutôt le processus technique qui imprime ses finalités à l'agir humain. Comme le dit Marx dans sa critique du machinisme, avec le développement de la technique capitaliste, les travailleurs sont devenus objets d'un procès qui s'est lui-même érigé en Sujet :

[...] c'est seulement avec la machinerie que ce renversement acquiert une réalité techniquement tangible, c'est pendant le procès même de travail que le moyen de travail, du fait de sa transformation en un automate, se pose face au travailleur comme capital, comme travail mort qui domine et aspire la force vivante du travail (1993 [1867]), p. 475).

Dans la section suivante, nous verrons que la dissolution de la médiation politique incarnée par l'État dans les sociétés modernes ne signifie pas tant la dissolution de la totalité comme le prétend la théorie des acteurs-réseaux en sociologie ou celle des usages en communication, mais plutôt la mise en place graduelle de la logique totalisante de la marchandise.

\section{Idéologie invisible et dissolution de la médiation politique}

À partir des postulats théoriques que nous avons étayés plus haut, il est possible de soutenir que la médiation des rapports sociaux au moyen des réseaux sociotechniques consiste en une forme fétichisée de rapport social de 
Médias sociaux, idéologie invisible et réel : pour une dialectique du concret

la même manière que Marx voyait dans la marchandise un rapport social inversé, c'est-à-dire "comme rapport impersonnel entre des personnes et rapports sociaux entre des choses impersonnelles » (Marx, 1993 [1867], pp. 8384). Comme le souligne Quéré: "Les médias assurent dans la société moderne la gestion du tiers symbolisant propre à une société historique. " (Quéré, 1982, p. 42). Quéré soutient notamment que les techniques de communication participent d'une mutation du mode de symbolisation des sociétés modernes avancées. Ce nouveau mode de symbolisation et d'objectivation se caractérise par ce que Claude Lefort nomme l'« idéologie invisible » (Lefort, 1978). Celle-ci est qualifiée d'invisible puisqu'elle apparaît comme n'étant pas idéologique, mais bien technique. Elle vise à estomper les divisions socio-temporelles et les antagonismes sociaux puisqu'elle repose sur un discours décrivant une société réconciliée avec elle-même. Elle prétend qu'il est possible d'accéder directement au réel, notamment grâce aux réseaux de communication. À l'instar de Lefort nous n'entendons pas la notion d'idéologie comme une forme délibérée de manipulation de la part d'une classe dominante puisque, comme nous le mentionnons plus haut, la dimension imaginaire du social est indissociable du réel.

Il est donc nécessaire d'identifier les différentes formes prises par l'idéologie au regard des transformations historiques des rapports sociaux. Afin que la médiation "automédiatisante " des techniques de communication s'insère comme lien social de substitution, il faut tout d'abord dissoudre les rapports d'interdépendance qui liaient les communautés pré-modernes. La croyance en une institution symbolique (Le Cosmos, les Esprits, le Peuple, la Nation, etc.), qui transcende la réalité strictement empirique de l'individualiste propriétaire (Macpherson, 1971), est au fondement de tout lien social. Ce travail de dissolution du rapport symbolique induit par la logique marchande résulte d'une dynamique historique. Si l'on suit l'analyse hégélienne, dans le monde moderne, la médiation politique a permis de suppléer, bien que de manière abstraite, la disparition des anciens rapports sociaux médiatisés symboliquement, notamment sous la forme de la communauté imaginée qu'est l'État-nation (Weil, 2002). L'État est donc venu suppléer au délitement des liens d'interdépendance directs induit par la médiation des rapports sociaux au moyen de la marchandise (Dufour, 2003).

II reste que dans la modernité, la tension instituée par la séparation du politique et de l'économique nécessite tout de même la production d'une idéologie qui vise à légitimer les contradictions de l'ordre social. Une des caractéristiques fondamentales de la modernité politique est que le pouvoir ne repose plus sur l'arbitraire, mais doit se légitimer au moyen de la médiation de 


\section{Maxime OUELLET, André MONDOUX et Marc MENARD}

l'idéologie pour assurer la reproduction de la société. L'idéologie n'est pas strictement négative ; elle est également positive, en ce qu'elle consiste en un discours qui s'inscrit à l'intérieur de l'espace public et qui agit comme moyen de légitimation des contradictions de l'ordre social (Côté, 1998). L'idéologie n'est pas qu'une perversion du réel (« une fausse conscience qui masque la réalité objective "), elle correspond plutôt à un ensemble de représentations dans lequel les sujets donnent un sens à leurs pratiques et à leurs relations sociales. II s'agit du mode d'expression et d'institutionnalisation politique du pouvoir dans les sociétés modernes. Bien qu'un antagonisme soit au fondement de la modernité politique, les dominants demeurent néanmoins soumis à une norme à partir de laquelle les divers groupes ou classes sociales jugent au moyen du débat public si elle est respectée ou non.

L'idéologie «bourgeoise " (Lefort, 1978) qui est au fondement de la modernité est travaillée par une contradiction fondamentale entre, d'une part, la capacité que se donne la société au moyen de la médiation politique d'agir réflexivement sur les conditions de sa propre historicité (Freitag, 1986) et, d'autre part, la domination croissante d'une forme autonomisée de pratique sociale qui s'érige comme une norme totalisante, c'est-à-dire l'économie. L'apparition de la catégorie du politique, en tant que médiation sociale constitutive de la modernité, même si elle demeure subsumée par la rationalité économique, rend possible l'émancipation humaine dans la mesure où elle institue un espace où se met en place une discussion sur les normes communes.

Selon Lefort, l'idéologie invisible surgit des contradictions de l'idéologie «bourgeoise " au fondement de la modernité. La principale contradiction de l'idéologie bourgeoise repose sur l'écart qu'elle introduit entre le réel et l'idéel : par exemple, entre l'idée de la liberté et la liberté réelle ou encore entre l'égalité formelle et l'égalité réelle. L'idéologie invisible prétend résoudre cette contradiction en améliorant la communication entre les individus dans la société au moyen de la technique. Elle apparaît dans la nouvelle configuration postlibérale du capitalisme où la régulation des rapports sociaux au moyen du marché est remplacée par la planification au sein des organisations (Galbraith, 1967). Au sein de ce capitalisme dit «avancé », le primat des rapports processuels aux dépens de l'institution symbolique incarnée par l'État conduit à l'effacement de la différenciation fonctionnelle entre les sphères de la pratique sociale (la culture, la politique, l'économie), lesquelles sont intégrées dans une communication de type autoréférentielle. La scission constitutive de la modernité entre État et société civile s'efface au profit d'une même logique communicationnelle-cybernétique partagée tant par les entreprises privées que 
Médias sociaux, idéologie invisible et réel : pour une dialectique du concret

par les institutions publiques (Freitag, 1986). Avec cette dissolution de la médiation politique incarnée par l'État, le capital est en mesure de se brancher directement sur l'espace public (Haber, 2012). Dans ce contexte, le discours social (celui de la vie quotidienne) absorbe le discours sur le social ('idéologie qui se veut en écart avec la réalité empirique). En d'autres termes, l'idéologie bourgeoise fusionne avec l'idéologie totalitaire dont la velléité était d'absorber l'ensemble de l'espace social dans un principe unique d'ordonnancement du réel. Selon Lefort, avec l'apparition de l'idéologie invisible, le réel devient idéologique lui-même, rendant caduque la séparation historique entre idéologie et réel. L'idéologie ne cherche plus à masquer l'écart entre la norme symbolique et le réel, elle prétend plutôt parler au nom du réel. À la manière de l'idéologie totalitaire, l'idéologie invisible est donc en mesure d'estomper les divisions fonctionnelles de l'idéologie bourgeoise (société civile/État, privé/public, interne/externe, etc.). Comme l'idéologie bourgeoise, elle se présente comme non-totalisante, malgré qu'elle s'apparente à un totalitarisme de la transparence communicationnelle qui vient anéantir la distinction entre espace privé et public. Comme nous le verrons dans la section suivante, l'idéologie invisible qui substitue la notion de totalité par celle de réseaux s'incarne parfaitement dans les médias socionumériques.

\section{Médias sociaux, idéologie invisible et surveillance}

À la dissolution du politique dans l'économique propre au capitalisme avancé correspond la disparition de la figure bourgeoise du sujet, c'est-à-dire l'individu en mesure de transcender ses intérêts particuliers afin de discuter des finalités sociétales au sein d'un espace public (Habermas, 1993). En clair, lorsque la figure de l'homme public disparaît (Sennett, 1979), l'homme économique dévore le sujet politique. II faut donc se poser la question de savoir en quoi la médiation des rapports sociaux au moyen des techniques de communication, notamment via les médias socionumériques, s'inscrit-telle dans cette nouvelle idéologie invisible et comment participe-t-elle à la reproduction de la subjectivité adaptée à cet ordre social ?

Il apparaît nécessaire de consulter la littérature sociologique qui a analysé l'émergence de nouvelles formes de subjectivité dans les sociétés capitalistes avancées. Christopher Lash (1980) a qualifié la figure idéal-typique qui émerge au sein de cette forme sociale de sujet narcissique ${ }^{5}$. Le sujet narcissique se

\footnotetext{
${ }^{5}$ Comme le souligne Stiegler, il ne faut pas comprendre le concept de narcissisme d'un point de vue psychologique: "c'est au contraire le système industriel de captation, de canalisation et de
} 


\section{Maxime OUELLET, André MONDOUX et Marc MENARD}

caractérise par son incapacité à distinguer le moi et le monde, il s'agit d'un individu privatisé. Les objets extérieurs lui apparaissent comme une projection de son propre moi :

«Le narcissisme c'est l'abolition de tout milieu distinct de la sphère du moi, de telle sorte que tout dans la société est miroir de la personnalité, qu'il n'y a plus aucune dimension sociale indépendante des désirs, des besoins et des capacités d'action présumée des sujets sociaux. D'une certaine manière la société intimiste $[\ldots]$ tend à réaliser le projet de toute utopie, qui est de renverser la tyrannie des médiations rigidifiées qui permettraient l'échange social (Dieu, État, monnaie, concepts, conventions); c'est-à-dire de parvenir à un monde sans représentation, un monde de la transparence dans laquelle la seule médiation acceptée est celle de la technique » (Quéré, 1982, p. 59).

Les médias socionumériques tels que Facebook constituent un exemple probant de ce type de subjectivité qui se prétend émancipée des médiations sociales et politiques et de ses institutions perçues comme hiérarchiques et autoritaires. En se présentant comme des lieux d'expression de soi, ils prétendent permettre à l'individu de construire son identité à la carte (Mondoux, 2011). Non seulement ils sont révélateurs d'une société intimiste (Sennett, 1979) où les distinctions entre le privé et le public disparaissent au profit d'un " entre-nous » (Lefort, 1978) au sein duquel les rapports horizontaux s'opposent aux rapports hiérarchiques des institutions, mais ils participent également à la reproduction d'une subjectivité adaptée à la société marchande. Ainsi, Facebook offre à ses abonnés de se définir par la création d'un profil misant principalement sur la consommation de biens culturels (livres, disques, émissions de télévision favoris), contribuant du coup à faire du consommateur et non plus le citoyen - l'étalon du vivre-ensemble. Chez le sujet narcissique, on retrouve la même perte du réel, la même absence d'un monde commun qui caractérise le fétichisme de la marchandise. Bref, le sujet narcissique est un individu délié, un pur sujet sans objet (Fischbach, 2011), puisque l'échange marchand n'est possible que dans une société où la rationalité calculatrice remplace les normes symboliques au fondement de la représentation d'un monde commun, incarnée notamment par l'idée de Cosmos (Foessel, 2012). Bien qu'il apparaisse comme libre, l'individu délié demeure soumis aux

déformation du désir, de l'investissement, de la sublimation et donc de la raison qui créé et exploite ce narcissisme » (2012, p. 66). 
Médias sociaux, idéologie invisible et réel : pour une dialectique du concret

injonctions de la dynamique abstraite d'auto-valorisation du capital sur laquelle il n'a aucune prise politiquement.

Ce type de subjectivité hyperindividualiste (Mondoux, 2011) est reproduit notamment par les dynamiques de surveillance mutuelle que l'on retrouve dans les médias sociaux. Inscrite à même la production du lien social, la surveillance devient ainsi banalisée : avec l'intégration de la géolocalisation au sein des médias socionumériques, dire en temps réel où l'on se situe fait désormais partie des outils d'auto-expression de soi. Rappelons que le panoptique conçu par Bentham, puis repris par Foucault comme l'illustration du pouvoir moderne, visait à mettre en place un dispositif institutionnel permettant de produire des acteurs rationnels qui calculent efficacement l'ensemble de leurs activités (Laval, 2007). Selon Bentham, la transparence complète des individus est essentielle pour instituer un sujet utilitariste; les citoyens de la société de marché doivent se rendre visibles et être en mesure de voir les autres afin de pouvoir évaluer, comptabiliser, et mesurer leur valeur et celle d'autrui. Comme le souligne Christian Laval, la formule de la nouvelle normativité propre à la société de marché c'est moins de «transcendance plus de surveillance » (Ibid., p. 258). Bref, la société de marché correspond davantage à la figure de la " chaîne invisible " inventée par Bentham qu'à la «main invisible " d'Adam Smith (Ibid.).

C'est pourquoi Bentham accorde également une importance primordiale à la communication en ce qu'elle se définit principalement comme un médium de transmission des informations. Faisant fi de sa dimension symbolique, on applique au langage les règles de l'efficience, c'est-à-dire que la valeur du langage est mesurée en fonction des effets qu'il produit. Ainsi les abonnés de Facebook sont-ils invités à jauger leurs communications selon la quantité d'amis qu'ils ont et le nombre de clics du bouton « j'aime " accumulés, transformant les «amis » en public des publicités qui se greffent aux contenus partagés.

L'exemple du mécanisme panoptique auquel on peut associer les médias socionumériques montre que, sans la médiation d'une méta-communication en mesure de transcender la réalité strictement empirique des individus (Quéré, 1982), c'est-à-dire d'une institution symbolique qui confère a priori un sens à la pratique sociale, la communication ne communique rien. Dans la société de marché, la communication tout comme le travail est aliéné (Agamben, 1990). À la manière du travail concret qui est subsumé par le travail abstrait dans le but de produire de la valeur, le langage n'est plus l'expression de l'expérience humaine sensible au fondement de la culture et de la réflexivité humaine, il est réifié. Le langage devient performatif (Lyotard, 1979) : la valeur de ce qui est 


\section{Maxime OUELLET, André MONDOUX et Marc MENARD}

communiqué ne se mesure pas en fonction de son contenu, de sa valeur d'usage, mais de sa valeur d'échange (Dean, 2002). La publicité de soi qu'encouragent les médias socionumériques illustre ce rôle performatif du langage dans les sociétés capitalistes avancées. En effet, les adeptes du « 2.0 » sont invités à faire le « branding » de leur personne, c'est-à-dire veiller à l'image de marque commerciale de leur présence au sein des médias socionumériques, évoquant ainsi le moyen de communication de la "société totalement administrée » (Adorno et Horkeimer, 1974, p. 172).

Au regard des présupposés qu'on retrouve dans les discours théoriques portant sur les possibilités d'empowerment des médias socionumériques (Proulx, 2011), il faut se demander si la médiation des rapports sociaux par le biais de réseaux sociotechniques n'instituerait pas une rupture avec la modernité politique dans la mesure où elle se présente comme a-idéologique. Ne représenterait-elle pas le passage vers un ordre social post-politique (Zizek, 2004), où le pouvoir ne nécessiterait plus le recours à une norme ou à un idéal régulateur pour se légitimer autre que celui de la valeur économique ? De fait, la légitimation de l'ordre social dans le monde numérique semble reposer essentiellement sur les règles de l'efficience technique et de la capacité organisationnelle fondées sur le calcul input/output (Valentine, 2000), conforme en cela à la définition que faisait Heidegger de la technique moderne : "Peutêtre est-il une pensée plus sobre que le déferlement irrépressible de la rationalisation et l'emportement qu'est la cybernétique. C'est plutôt cet emportement qui pourrait bien être le comble de l'irrationnel. " (Heidegger, 1990, p. 138). II convient de rappeler, afin de ne pas tomber dans le piège du discours idéologique, qu'il s'agit bien dans les faits d'une nouvelle forme d'idéologie, d'une idéologie invisible, qui prend la forme essentiellement managériale et apolitique du contrôle (Deleuze, 1990). Une des formes idéalisées de ce contrôle est celle de la nature, modèle parfait de l'efficacité maximale et qui inspire plus d'un ténor du Web 2.0 :

«[...] la nature peut nous enseigner plusieurs leçons, comme le comportement des colonies de fourmis, qui ont inspiré les programmes informatiques de rationalisation des processus de fabrication, des réseaux téléphoniques et des itinéraires de camions. Les termites peuvent nous aider à trouver des solutions au contrôle climatique et les bancs de poissons ont montré aux militaires américains comment modéliser une équipe de robots. [...] Les hiérarchies sont typiquement bureaucratiques et les employés manquent de motivation. Elles sont de plus en plus insuffisantes comme 
Médias sociaux, idéologie invisible et réel : pour une dialectique du concret

moyen ou pour organiser le rythme rapide d'une économie où le capital humain doit être libéré pour l'innovation, la création de valeur et les relations avec la clientèle » (trad. libre, Tapscott, 2010, p. 1).

II ne s'agit pas ici de soutenir que la capacité de diffusion massive d'informations rendue possible par les médias sociaux ne permettrait pas aux militants de déjouer la propagande gouvernementale et celle des médias de masse traditionnels. Comme nous l'ont montré les exemples récents des mouvements de révolte de type Occupy ou encore du Printemps arabe, les médias sociaux ont contribué à la diffusion d'informations alternatives. Cependant, les analyses qui voient dans les médias sociaux l'apparition d'un nouvel espace public ${ }^{6}$ sont problématiques pour deux raisons principales. D'une part, elles souffrent d'une compréhension fétichisée de la technique, voyant dans les médias sociaux le facteur explicatif de la mobilisation sociale, comme en témoigne l'expression révolution Facebook (Fuchs, 2014). D'autre part, ce type d'analyse ne permet pas d'expliquer pourquoi ces révoltes qui ont surgi à la suite d'une des plus grandes crises que le capitalisme ait connues historiquement n'ont pas réussi à s'instituer politiquement? L'une des explications possibles repose sur le fait que ces mouvements refusent de s'identifier à toute forme d'idéologie. Comme le souligne le journaliste de la BBC Paul Mason dans son analyse des mouvements de révolte contemporain, ces mouvements "s'opposent à toutes les idéologies: travaillisme, islamisme, catholicisme... en fait toutes les formes d'idéologies hermétiques sont rejetées " (Mason, 2011). Selon lui, la seule idéologie qui rassemble les individus participant à ces mouvements est celle de l'horizontalisme du réseau, c'est-àdire une idéologie invisible qui se fonde sur la même ontologie sociale que le néolibéralisme ${ }^{7}$.

\section{Pour une dialectique du concret}

Nous avons soutenu dans cet article qu'il est essentiel de retourner aux notions fondamentales de la théorie critique afin d'appréhender des phénomènes comme les médias socionumériques dans leur totalité. Comme Granjon (2004), nous soutenons que l'attention unilatérale posée sur la dimension des pratiques concrètes dans plusieurs approches qui traitent des médias socionumériques, notamment celle des usages, tend à négliger la manière dont ceux-ci sont intégrés dialectiquement avec une structure de

\footnotetext{
${ }^{6}$ Voir par exemple Shirky, 2011; Gladwell et Shirky, 2011, et Mason 2012.

${ }^{7}$ À ce sujet voir Dean 2002, Labelle 2007, Mirowski 2013.
} 


\section{Maxime OUELLET, André MONDOUX et Marc MENARD}

domination abstraite. Nous ne croyons cependant pas comme ce dernier qu'il faille abandonner la théorie critique d'inspiration francfortoise en raison de son pessimisme (lbid.). Au contraire, les notions hégéliano-marxienne mobilisées par l'École de Francfort, comme celles de fétichisme, d'aliénation, de totalité et d'idéologie permettent d'élaborer une théorie dialectique du concret qui dépasse l'opposition entre les approches qui nient l'existence de la totalité pour tenter de redécouvrir les capacités d'agir des acteurs d'une part, et d'autre part les approches qui valorisent la totalité au détriment des acteurs. Comme l'observe Frédéric Vandenberghe :

"La distinction entre la forme et le contenu de la marchandise nous invite à prolonger l'analyse performative de la construction du réseau d'éléments visibles par des acteurs locaux par une analyse plus systématique des structures relationnelles qui surdéterminent la forme que peut prendre ou, le cas échéant, ne pas prendre l'assemblage socio-technique des éléments. L'introduction d'un méta-niveau de détermination concrète ne signifie pas pour autant que tout ce qui se passe localement est rigoureusement déterminé par des structures globales et englobantes, mais elle nous permet d'analyser comment, et jusqu'à quel point, les structures existantes de domination tendent à exclure l'émergence d'un ordonnancement alternatif des relations sociales entre humains, ainsi que des éléments hétérogènes qu'ils associent et assemblent dans un ensemble socio-technique " (Vandenberghe, 2006, p. 171).

En clair, la capacité d'agir des acteurs n'est pas opposée à la structure ; celles-ci sont liées dialectiquement puisque toute pratique s'inscrit dans un monde qui est déjà pré-structuré, et la structure ne peut se reproduire qu'à partir de l'action subjective. Le problème des approches qui priorisent l'agence ${ }^{8}$ est

\footnotetext{
${ }^{8}$ Par agence nous référons à la notion d'agency en théorie sociologique. Dans ce débat nous refusons la fausse opposition entre agent et structure au profit d'une approche dialectique selon laquelle la capacité d'agir des acteurs s'inscrit dans des structures qui sont produites et (re)produites par la pratique sociale. À ce sujet voir Pierre Bourdieu, 2000. On observe dans les relectures spinozistes de Marx depuis Althusser, en passant par Antonio Negri et plus récemment Frédéric Lordon (2010), un abandon des catégories dialectiques d'aliénation, de fétichisme et d'idéologie, au profit de la notion de capacité d'agir. Ce tournant non dialectique de la théorie critique pose problème selon nous puisque ces approches ne permettent pas de penser une sortie de la situation d'aliénation des sujets, pour autant qu'on ne pense pas l'aliénation comme une perte de la capacité d'affirmation subjective des individus, mais plutôt comme une incapacité de se réapproprier l'objectivité sociale. Ce type de lecture mène Hardt et Negri (2000) à soutenir que les nouvelles
} 
Médias sociaux, idéologie invisible et réel : pour une dialectique du concret

qu'elles tombent dans le piège empiriste qui repose sur une forme de " fétichisme du concret ». Ces approches sont incapables de voir que la réalité empirique est déterminée par un élément invisible que seule la méthode dialectique est en mesure de reconstruire conceptuellement. II ne s'agit pas de nier qu'il existe effectivement certaines pratiques au sein des médias sociaux qui sont en opposition avec la logique marchande, mais plutôt de montrer que ces pratiques qui relèvent d'une autre logique, comme celle du don qu'on retrouve dans le partage gratuit de certains contenus en ligne comme l'annotation (d'un document), l'indexation, la collaboration à la réalisation de documents collectifs, etc. (Proulx, 2011), assurent «le côté obscur de la valeur " sans lequel la société marchande ne pourrait se reproduire (Jappe, 2009). Plusieurs travaux en économie politique de la communication soutiennent par ailleurs que ces pratiques qui sont en apparence nonmarchandes, comme celle de la prosommation, sont de plus en plus subordonnés à la logique de valorisation du capital (Comor, 2010). Dans la culture du capitalisme communicationnel les individus qui sont de plus en plus « incapacités » par les nouvelles technologies doivent cependant devenir euxmêmes des marchandises afin de se subjectiver (Bauman, 2007), comme l'atteste la populaire tendance du «branding » consistant à " gérer » son image publique comme une marque de commerce :

"Le personal branding [sic] arrive en ville et beaucoup de gens commencent à se demander, dois-je vraiment? J'écris cet article pour les encourager à dire oui, oui, vous devez commencer à penser à votre personal branding. Votre avenir pourrait en être marqué. [...] On comprendra que le temps est venu au rayonnement personnel et de s'ouvrir aux réseaux sociaux et aux plateformes qui nous permettent de bâtir notre propre marque de commerce. ${ }^{9}$

Dans la mesure où le capital se nourrit de plus en plus de la désinstitutionalisation et favorise la collaboration horizontale des usagers pour assurer sa reproduction, la théorie critique se doit de remettre en question la figure libérale de la subjectivité et repenser les médiations et les institutions qui

technologies permettraient aux individus en réseaux de se passer de toute forme de médiation sociale afin de réaliser de manière spontanée un ordre social autorégulé, position qui se rapproche des thèses néolibérales sur les médias sociaux.

${ }^{9}$ Micheline Bourque, consultante en marketing, « Personal Branding, version Fabrice Calando (aka Hack Your Life)», blogue personnel, publié le 23 janvier 2013 à http://www.michelinebourque.com/2013/01/23/personal-branding-version-fabrice-calando-aka-hackyour-life/, dernière consultation, le 22/02/2013. 


\section{Maxime OUELLET, André MONDOUX et Marc MENARD}

sont les conditions ontologiques de la liberté individuelle et collective. II s'agit donc de rompre avec l'imaginaire libéral au fondement de la pensée moderne qui repose sur une fausse conception anthropologique. Son individualisme méthodologique oublie que l'individu ne peut se construire ni avant ni sans les autres (Généreux, 2006). Comme nous l'avons montré, l'illusion de l'ontologie libérale apparaît pleinement dans le désir des sujets déliés de s'exprimer dans les réseaux sociaux. Ce sujet pseudo-émancipé des institutions se retrouve paradoxalement dans la dépendance humiliante et constante du regard d'autrui afin d'exister (Labelle, 2007). Contrairement à ce que prétend l'idéologie libérale, les humains sont des êtres sociaux qui ont besoin des institutions afin de s'individualiser :

«II n’y a ni comme réalité, ni comme fiction cohérente d'individu humain comme substance a-, extra, ou présociale. Nous ne pouvons concevoir un individu sans langage, par exemple, et il n'y a de langage que comme création et institution sociale. Cette création et cette institution ne peuvent pas être vue, sans ridicule, comme résultant d'une coopération délibérée des individus - ni d'une addition de réseaux intersubjectifs: pour qu'il y ait intersubjectivité, il faut qu'il y ait des sujets humains et la possibilité qu'ils communiquent - autrement dit, des êtres humains déjà socialisés et un langage qu'ils ne sauraient produire eux-mêmes en tant qu'individus (un ou plusieurs réseaux intersubjectifs), mais doivent recevoir de leur socialisation » (Castoriadis, 1996, p. 222).

Ce que dit Castoriadis à propos de l'institution imaginaire de la société et du type de subjectivité qui lui correspond s'applique également à la technique. Les techniques de communication modernes sont la concrétisation dans la pratique des idéaux de la modernité capitaliste. La demande d'autonomie et d'autoréalisation de même que la capacité de se dégager, de se délier, et le pouvoir de se réengager et de se relier tout aussi librement, sont réalisés de manière effective dans l'usage de ces médias. Plutôt que d'une réelle libération, il faut voir là la forme la plus adéquate de l'aliénation puisqu'elle implique une dissolution du politique, c'est-à-dire l'impossibilité d'instituer des valeurs communes partagées socialement. L'aliénation, comme le rappelle Frank Fishbach (2011), c'est la perte du monde commun, et les médias sociaux participent pleinement à la dissolution de ces normes communes. Nul exemple plus révélateur que celui des milliers d'abonnés à Facebook qui, mécontents de la prolifération des mini-applications au sein du service, n'arrivent pourtant pas à 
Médias sociaux, idéologie invisible et réel : pour une dialectique du concret

unir leurs forces en une seule voix et se manifestent en centaines de groupuscules individuels, mais néanmoins tous portés vers la même cause. De fait, la restructuration des rapports sociaux sous une forme réticulaire induit un repli sur soi dans lequel des identités choisissent, comme dans un marché, leurs propres communautés d'appartenance. Ce repli communautariste engendre un déni du politique, puisqu'il repose sur le refus du rapport d'altérité au fondement du lien social. En retour, ce refus des diverses communautés d'appartenances identitaires d'entrer en rapports politiques les unes avec les autres reproduit les structures de la société de marché. Comme le signalait Simondon :

"L'automate est communautaire, et non individualisé comme un être vivant capable de se mettre en question luimême. Une communauté pure se conduirait comme un automate; elle élabore un code de valeurs destinées à empêcher les changements de structure, et à éviter la position des problèmes. Les sociétés au contraire, qui sont des groupements synergiques d'individus, ont pour sens de chercher à résoudre des problèmes. Elles mettent en question leur propre existence, tandis que les communautés cherchent à persévérer dans leur être. Norbert Wiener a analysé la manière dont les pouvoirs de rigidité d'une communauté assurent son homéostasie. La communauté tend à automatiser les individus qui la composent, en leur donnant une signification fonctionnelle pure. » (2005, p. 519).

\section{Références Bibliographiques}

ADORNO T. et M. HORKHEIMER, 1974, La dialectique de la raison, Paris, Gallimard.

AXELOS K., 1961, Marx Penseur de la technique, Paris, Les Éditions de minuit.

AGAMBEN G., 1990, " Gloses marginales aux commentaires sur la société du spectacle ", Multitudes, http://www.multitudes.samizdat.net/Gloses-marginalesaux-Commentaires, dernière consultation le 22 décembre 2012.

BAUMAN Z., 2007, Consuming Life, London, Polity.

BOURDIEU P., 2000, Esquisse d'une théorie de la pratique, Paris, Seuil. 


\section{Maxime OUELLET, André MONDOUX et Marc MENARD}

CASTELLS M., 2001, L'ère de l'information. Vol I : La société en réseaux, Paris, Fayard.

CASTORIADIS C., 1975, L'institution imaginaire de la société, Paris, Seuil.

CASTORIADIS C., 1996, "La démocratie comme procédure et comme régime », dans Les Carrefours du labyrinthe IV : la montée de l'insignifiance, Paris, Seuil.

COMOR E., 2010, «Contextualizing and Critiquing the Fantastic Prosumer: Power, Alienation and Hegemony », Critical Sociology, vol. 37, n³, pp. 309-327.

CÔTÉ J.-F., 1998, « La société de communication à la lumière de la sociologie de la culture », Sociologie et sociétés, vol. 30, n¹, pp. 1-16.

DEAN J., 2002, Publicity's secret: How technoculture capitalizes on democracy, Ithaca, Cornell University Press.

DELEUZE G., 1990, «Post-scriptum sur les sociétés de contrôle », L'autre journal, $\mathrm{n}^{\circ} 1$, http://infokiosques.net/lMG/pdf/Deleu.pdf, dernière consultation le 22 février 2013.

DUFOUR D.-R., 2003, "Conjectures sur le social et les sciences sociales ", Revue européenne des sciences sociales, Tome XLI, n¹27, pp. 51-62.

FISCHBACH F., 2009, Manifeste pour une philosophie sociale, Paris, La Découverte.

FISCHBACH F., 2011, La privation du monde : Temps, espace et capital, Paris, Vrin.

FOESSEL M., 2012, Après la fin du monde : critique de la raison apocalyptique, Paris, Seuil.

FREITAG M., 1986, Dialectique et société, Tome 2, Montréal, Éditions Saint-Martin.

FREITAG M., 2008, L'impasse de la globalisation, Montréal, Écosociété.

FUCHS C., 2014, Social media. A Critical introduction, London, Sage.

GALBRAITH J. K., 1967, The New Industrial State, Boston, Houghton Mifflin.

GÉNÉREUX J., 2006, La dissociété, Paris, Seuil.

GLADWELL M. et C. SHIRKY, 2011, «From innovation to revolution : Do social media make protests possible ? ", Foreign Affairs, vol. 90, n², pp. 153-154.

GOUX J.-J., 1973, Freud, Marx. Économie et symbolique, Paris, Éditions du Seuil.

GRANJON F., 2004, « De quelques éléments programmatiques pour une sociologie critique des usages sociaux des TIC », dans S. HADJ-ALI (dir.), Les 
Médias sociaux, idéologie invisible et réel : pour une dialectique du concret rapports société-technique du point de vue des sciences de l'homme et de la société, http://archivesic.ccsd.cnrs.fr/docs/00/06/24/54/PDF/sic 00001155.pdf, dernière consultation le 15 janvier 2012.

HABER S., 2012, « Un espace public néocapitaliste ? Habermas, un demi-siècle après ", Variations, $n^{\circ} 16$, http://variations.revues.org/204, dernière consultation le 22 février 2012.

HABERMAS J., 1993, L'espace public : Archéologie de la publicité comme dimension constitutive de la société bourgeoise, Paris, Payot.

HARDT M. et A. NEGRI, 2000, Empire, Paris, Exils.

HEIDEGGER M., 1990. Questions III et IV, Paris, Gallimard.

HORKHEIMER M., 1970, Théorie traditionnelle et théorie critique, Paris, Gallimard.

JAURÉGUIBERRY F. et S. PROULX, 2011, Usages et enjeux des technologies de communication, Toulouse, Erès.

JAPPE A., 2009, «Le « côté obscur » de la valeur et le don », Revue du Mauss, n॰34, pp. 96-113.

KOSIK K., 1988, La dialectique du concret, Paris, Les éditions de la passion.

LABELLE G., 2007, «Essai sur l'idéologie dominante de la démocratie avancée (ou : à quoi sert le différentialisme?) ", dans S. VIBERT (dir), Pluralisme et démocratie. Entre culture, droit et politique, Montréal, Québec Amérique, pp. 103-125.

LASCH C., 1980, Le Complexe de Narcisse : la nouvelle sensibilité américaine, Paris, Robert Laffont.

LAVAL C., 2007, L'homme économique : essai sur les racines du néolibéralisme, Paris, Gallimard.

LEFORT C., 1978, Les formes de l'histoire : essais d'anthropologie politique, Paris, Gallimard.

LORDON F., 2010, Capitalisme, désir et servitude. Marx et Spinoza, Paris, La Fabrique.

LYOTARD J.-F., 1979, La condition postmoderne : rapport sur le savoir, Paris, Éditions de Minuit.

MACPHERSON C.B., 1971, La théorie politique de l'individualisme possessif : de Hobbes à Locke, Paris, Gallimard. 


\section{Maxime OUELLET, André MONDOUX et Marc MENARD}

MARX K., 1857, Introduction à la critique de l'économie politique, http://www.marxists.org/francais/marx/works/1857/08/km18570829.htm, dernière consultation le 22 février 2013.

MARX K., 1993 [1867], Le Capital, Livre 1, Paris, Presses universitaires de France.

MONDOUX A. et J.-G. LACROIX, 2009, «Fausse et vraie diversité culturelle dans le système-monde ", dans Y. THEORET (dir), David contre Goliath : La Convention sur la protection et la promotion de la diversité des expressions culturelles de l'UNESCO, Éditions Hurtubise, Montréal.

MASON P., 2011, “Twenty reasons why it's kicking off everywhere ». Iddle Scrawl. Paul Mason's blog, BBC, Saturday 5, 2011. En ligne : http://www.bbc.co.uk/blogs/newsnight/paulmason/2011/02/twenty reasons why its kicking.html, dernière consultation le 30 janvier 2014.

MASON P., 2012, Why it's kicking off everywhere. The new global revolutions, London, Verso.

MONDOUX A., 2011, «Identité numérique et surveillance », Les cahiers du numérique, $\mathrm{n}^{\circ} 1$, pp. 1-13.

MONDOUX A., 2012a, «Technique et individuation : la part du social », dans C. PERRATON, O. KANE et F. DUMAIS (dir), Mobilisation de l'objet technique dans la production de soi, Presses de l'Université du Québec, collection Cahiers du Gerse, pp. 37-56.

MONDOUX A., 2012b, « À propos du social dans les médias sociaux », Terminal, Paris.

LATOUR B., 2007, « Une sociologie sans objet ? Note théorique sur l'interobjectivité ", dans O. DEBARY (dir), Objets et mémoires, Québec, Presses de l'Université Laval, pp. 38-57.

POSTONE M., 2009, Temps, travail et domination sociale, Paris, Mille et une nuits.

PROULX S., 2011, « La puissance d'agir d'une culture de la contribution face à l'emprise d'un capitalisme informationnel. Premières réflexions ", Revue du MAUSS permanente, http://www.journaldumauss.net/spip.php?article825, dernière consultation le 13 janvier 2013.

QUÉRÉ L., 1982, Des miroirs équivoques : aux origines de la communication moderne, Paris, Aubier-Montaigne.

SENNETT R., 1979, Les tyrannies de l'intimité, Paris, Le Seuil.

SHIRKY C., 2011, « The political power of social media », Foreign Affairs, vol. 90, n०1, pp. 28-41. 
Médias sociaux, idéologie invisible et réel : pour une dialectique du concret

SIMONDON G., 2005, L'individuation à la lumière des notions de forme et d'information, Paris, Jérôme Million.

STIEGLER B., 2004, De la misère symbolique, 1. L'époque hyperindustrielle, Paris, Galilée.

STIEGLER B., 2012, États de choc. Bêtise et savoir au XXe siècle, Paris, Mille et une nuits.

TAPSCOTT D., 2010, What nature teaches us about building better human organizations, http://dontapscott.com/2010/08/13/what-nature-teaches-us-aboutbuilding-better-human-organizations/, dernière consultation le 28 novembre 2012.

TRAN H. H., 2003, Relire le Capital, Paris, Page Deux Éditions.

VALENTINE J., 2000, «Information Technology, Ideology and Governmentality », Theory, Culture \& Society, vol. 17, n², pp. 21-43.

VANDENBERGHE F., 2006, Complexités du posthumanisme : trois essais dialectiques sur la sociologie de Bruno Latour, Paris, L'Harmattan.

VIOULAC J., 2009, L'époque de la technique. Marx, Heidegger et l'accomplissement de la métaphysique, Paris, Presses universitaires de France.

VIOULAC J., 2013, La logique totalitaire. Essai sur la crise de l'Occident, Paris, Presses universitaires de France.

VOIROL O., 2008, « Médiations et théorie critique. Questions et actualité d'un projet sociologique », Réseaux, vol. 2, n¹48-149, pp. 47-78.

WEIL É., 2002, Hegel et l'État : cinq conférences, Paris, Vrin.

ZIZEK S., 2004, Plaidoyer en faveur de l'intolérance, Paris, Climats. 